\title{
On certain biquadratic equations
}

\author{
A. Schinzel AND M. SkałBA
}

\begin{abstract}
It is proved that if certain biquadratic equations with a prime parameter and four unknowns have a non-trivial solution in the integers, then they have infinitely many such solutions.
\end{abstract}

Keywords. Pell equation, Biquadratic Diophantine equation, Cyclotomic polynomial.

The diophantine equation $f(x, y, z, t)=c$, where $f$ is a quartic form and $c \neq 0$, has been studied only in the case where $f$ splits over the complex field. The aim of this paper is to prove the following theorems.

Theorem 1. If $p \equiv 3(\bmod 4)$ is a prime, $\alpha \equiv 1(\bmod 2), D=p^{\alpha}$, and the equation $\left(x^{2}+y^{2}\right)^{2}-D\left(z^{2}+t^{2}\right)^{2}=1$ has at least one integer solution with $z^{2}+t^{2}>0$, then it has infinitely many integer solutions.

Theorem 2. If $p \equiv 3(\bmod 8)$ is a prime, $\alpha \equiv 1(\bmod 2), D=p^{\alpha}$, and the equation $\left(x^{2}+y^{2}\right)^{2}-D\left(z^{2}+t^{2}\right)^{2}=-2$ has at least one integer solution, then it has infinitely many.

Theorem 3. If $p \equiv 7(\bmod 8)$ is a prime, $\alpha \equiv 1(\bmod 2), D=p^{\alpha}$, and the equation $\left(x^{2}+y^{2}\right)^{2}-D\left(z^{2}+t^{2}\right)^{2}=2$ has at least one integer solution, then it has infinitely many.

It is natural to ask about the number $N_{i}(x)$ of values $D<x$ covered by the above Theorem $i(i=1,2,3)$. We cannot prove that $\lim _{x \rightarrow \infty} N_{i}(x)=\infty$, but since Theorem 2 and 3 apply to all odd primes of the form $\left(x^{2}+y^{2}\right)^{2} \pm$ 2 and Theorem 1 applies to all odd primes of the form $\left(x^{2}+y^{2}\right)^{2}+2$ at least heuristically $N_{i}(x) \gg \frac{x^{1 / 2}}{(\log x)^{3 / 2}}(i=1,2,3)$. The method does not seem applicable to other equations $\left(x^{2}+y^{2}\right)^{2}-D\left(z^{2}+t^{2}\right)^{2}=c$, where $c \neq 0$.

Lemma 1. Let $n$ be an odd, square-free integer $>3$ and $\Phi_{n}(x)$ the $n$-th cyclotomic polynomial. Then $\Phi_{n}(z)$ can be written in the form

$$
4 \Phi_{n}(z)=A_{n}(z)^{2}-(-1)^{(n-1) / 2} n z^{2} B_{n}(z)^{2},
$$


where $A_{n}(z)$ and $B_{n}(z)$ have integer coefficients and are of degree $\phi(n) / 2$ and $\phi(n) / 2-2$, respectively. $A_{n}(z)$ is symmetric if its degree is even, otherwise it is anti-symmetric. $B_{n}(z)$ is symmetric for $n$ prime.

Proof. See [[1], p.330 and 445].

Lemma 2. For $p \equiv 3(\bmod 4)$ a prime, $p>3, A_{p}(z)$ is divisible by $z-1$ and $B_{p}(z)$ is divisible by $z+1$.

Proof. We have [[1], p.330]

$$
\begin{aligned}
& A_{p}(z)+\sqrt{-p} B_{p}(z)=2 \prod_{\left(\frac{r}{p}\right)=1}\left(z-\zeta_{p}^{r}\right), \\
& A_{p}(z)-\sqrt{-p} B_{p}(z)=2 \prod_{\left(\frac{s}{p}\right)=-1}\left(z-\zeta_{p}^{s}\right) .
\end{aligned}
$$

If $p \equiv 3(\bmod 4)$ is a prime, then $\left(\frac{r}{p}\right)=1$ is equivalent to $\left(\frac{-r}{p}\right)=-1$. Since for $p>3, \sum_{\left(\frac{r}{p}\right)=1} r \equiv 0(\bmod p)$, we obtain

$$
\begin{gathered}
\prod_{\left(\frac{s}{p}\right)=-1}\left(1-\zeta_{p}^{s}\right)=\prod_{\left(\frac{r}{p}\right)=1}\left(1-\zeta_{p}^{-r}\right)=-\prod_{\left(\frac{r}{p}\right)=1}\left(1-\zeta_{p}^{r}\right) \\
\prod_{\left(\frac{s}{p}\right)=-1}\left(-1-\zeta_{p}^{s}\right)=\prod_{\left(\frac{r}{p}\right)=1}\left(-1-\zeta_{p}^{-r}\right)=\prod_{\left(\frac{r}{p}\right)=1}\left(1-\zeta_{p}^{r}\right)
\end{gathered}
$$

thus $A_{p}(1)=B_{p}(-1)=0$ and the lemma follows.

Lemma 3. If a form $F \in \mathbb{Q}[x, y]$ satisfies $F(x, y)=F(y, x)$ and $d:=\operatorname{deg} F \equiv 0$ $(\bmod 2)$, then $F=G\left((x+y)^{2}, x y\right)$, where $G \in \mathbb{Q}[z, t]$.

Proof. By the theorem on symmetric functions $F=H(x+y, x y)$, where $H \in$ $\mathbb{Q}[z, t]$. Let $F(x, y)=\sum a_{\alpha \beta}(x+y)^{\alpha}(x y)^{\beta}$. Since for $a_{\alpha \beta} \neq 0$ we have $\alpha+2 \beta=$ $d \equiv 0(\bmod 2)$, we have $a_{\alpha \beta}=0$ for $\alpha$ odd.

Lemma 4. Let $D=p^{\alpha}$, where $p \equiv 3(\bmod 4)$ is a prime, $\alpha \equiv 1(\bmod 2)$, and positive integers $u, v$ satisfy $u^{2}-D v^{2}=1$. Put

$$
\xi=u+v \sqrt{D}, \xi^{-1}=u-v \sqrt{D} ; L_{n}=\frac{\xi^{n}-\xi^{-n}}{\xi-\xi^{-1}},
$$

and

$$
E_{n}=\frac{\xi^{n}+\xi^{-n}}{2}, F_{n}=v L_{n}
$$

$E_{n}$ and $F_{n}$ are positive integers satisfying

$$
E_{n}^{2}-D F_{n}^{2}=1
$$

Moreover, for all positive integers $n$,

$$
E_{p^{n}} / E_{p^{n-1}} \text { is a sum of two squares. }
$$


Proof. We have

$$
E_{n}^{2}-D F_{n}^{2}=\frac{\xi^{2 n}+2+\xi^{-2 n}}{4}-D v^{2} \frac{\xi^{2 n}-2+\xi^{-2 n}}{4 v^{2} D}=1,
$$

which proves (3). Moreover,

$$
E_{p^{n}} / E_{p^{n-1}}=\Phi_{2 p^{n}}\left(\xi, \xi^{-1}\right)=\Phi_{2 p}\left(\xi^{p^{n-1}}, \xi^{-p^{n-1}}\right)=\Phi_{p}\left(-\xi^{p^{n-1}}, \xi^{-p^{n-1}}\right),
$$

where $\Phi_{n}(x, y)$ is a homogeneous form of $\Phi_{n}(z)$. By Lemma 1 for $p>3$

$$
4 \Phi_{p}\left(-\xi^{p^{n-1}}, \xi^{-p^{n-1}}\right)=A_{p}\left(-\xi^{p^{n-1}}, \xi^{-p^{n-1}}\right)^{2}+p B_{p}\left(-\xi^{p^{n-1}}, \xi^{-p^{n-1}}\right)^{2} .
$$

Since $A_{p}$ is anti-symmetric, $A_{p}\left(-\xi^{p^{n-1}}, \xi^{-p^{n-1}}\right)$ is symmetric in $\xi, \xi^{-1}$, hence is an integer. Now $B_{p}(x, y)$ is, by Lemma 2 , divisible by $x+y$, and the quotient is of degree $\frac{p-1}{2}-3 \equiv 0(\bmod 2)$. By Lemma 3 the quotient is a polynomial in $(x+y)^{2}$ and $x y$. It follows that $B_{p}\left(-\xi^{p^{n-1}}, \xi^{-p^{n-1}}\right)^{2}=p w^{2}$ with $w \in \mathbb{Q}$.

(4) follows now from (5) and (6). It remains to consider $p=3$. Let $\xi^{3^{n-1}}=$ $u_{n}+v_{n} \sqrt{3^{\alpha}}$. We have by (5)

$$
\begin{aligned}
E_{3^{n}} / E_{3^{n-1}} & =\Phi_{3}\left(-\xi^{3^{n-1}}, \xi^{-3^{n-1}}\right) \\
& =\left(u_{n}+v_{n} \sqrt{3^{\alpha}}\right)^{2}-\left(u_{n}+v_{n} \sqrt{3^{\alpha}}\right)\left(u_{n}-v_{n} \sqrt{3^{\alpha}}\right)+\left(u_{n}-v_{n} \sqrt{3^{\alpha}}\right)^{2} \\
& =u_{n}^{2}+\left(3^{\frac{\alpha+1}{2}} v_{n}\right)^{2} .
\end{aligned}
$$

Lemma 5. In the notation of Lemma 4,

$$
F_{p^{n}} / p F_{p^{n-1}} \text { is a sum of two squares. }
$$

Proof We have by (1) and (2)

$$
F_{p^{n}} / F_{p^{n-1}}=\Phi_{p^{n}}\left(\xi, \xi^{-1}\right)=\Phi_{p}\left(\xi^{p^{n-1}}, \xi^{-p^{n-1}}\right)
$$

and by Lemma 1 ,

$$
4 F_{p^{n}} / F_{p^{n-1}}=A_{p}\left(\xi^{p^{n-1}}, \xi^{-p^{n-1}}\right)^{2}+p B_{p}\left(\xi^{p^{n-1}}, \xi^{-p^{n-1}}\right)^{2} .
$$

Since $A_{p}(x, y)$ is anti-symmetric, $A_{p}(x, y) /(x-y)$ is symmetric and

$$
A_{p}\left(\xi^{p^{n-1}}, \xi^{-p^{n-1}}\right)^{2}=p w^{2}, w \in \mathbb{Q} .
$$

Also $B_{p}(x, y)$ is symmetric, $B_{p}\left(\xi^{p^{n-1}}, \xi^{-p^{n-1}}\right)$ is an integer, and (7) follows from (9) and (10). It remains to consider $p=3$. Let again $\xi^{3^{n-1}}=u_{n}+v_{n} \sqrt{3^{\alpha}}$. We have by $(8)$

$$
\begin{aligned}
F_{3^{n}} / 3 F_{3^{n-1}}= & \frac{1}{3}\left(\left(u_{n}+v_{n} \sqrt{3^{\alpha}}\right)^{2}+\left(u_{n}+v_{n} \sqrt{3^{\alpha}}\right)\left(u_{n}-v_{n} \sqrt{3^{\alpha}}\right)\right. \\
& \left.+\left(u_{n}-v_{n} \sqrt{3^{\alpha}}\right)^{2}\right) \\
= & u_{n}^{2}+\left(3^{\frac{\alpha-1}{2}} v_{n}\right)^{2} .
\end{aligned}
$$

Proof of Theorem 1. It follows by induction on $n$ from Lemmas 4 and 5 that for $n$ even $E_{p^{n}}$ and $F_{p^{n}}$ are sums of two squares. The formula (3) gives the theorem. 
Lemma 6. Let $\varepsilon= \pm 1, D=p^{\alpha}$, where $p \equiv 5+2 \varepsilon(\bmod 8)$ is a prime, $\alpha \equiv 1$ (mod 2), and positive integers $u, v$ satisfy $u^{2}-D v^{2}=2 \varepsilon$. Put

$$
\eta=\frac{\sqrt{2 \varepsilon} u+\sqrt{2 D \varepsilon} v}{2}, \eta^{-1}=\frac{\sqrt{2 \varepsilon} u-\sqrt{2 D \varepsilon} v}{2}, P_{n}=\frac{\eta^{n}-\eta^{-n}}{\eta^{(n, 2)}-\eta^{-(n, 2)}},
$$

and

$$
G_{n}=\frac{\eta^{n}+\eta^{-n}}{\sqrt{2 \varepsilon}}, H_{n}=v P_{n} .
$$

$G_{n}$ and $H_{n}$ are rational integers satisfying

$$
G_{n}^{2}-D H_{n}^{2}=2 \varepsilon
$$

Moreover, for all positive integers $n$,

$$
G_{p^{n}} / \varepsilon G_{p^{n-1}} \text { is a sum of two squares. }
$$

Proof. We have for odd $n$

$$
G_{n}^{2}-D H_{n}^{2}=\frac{\eta^{2 n}+2+\eta^{-2 n}}{2 \varepsilon}-D v^{2} \frac{\eta^{2 n}-2+\eta^{-2 n}}{2 \varepsilon D v^{2}}=\frac{4}{2 \varepsilon}=2 \varepsilon,
$$

which proves (13). $P_{n}$ as a Lehmer number is a rational integer, so is $H_{n}$ and, by $(13), G_{n}$ is an algebraic integer. However, it is also rational being invariant to all automorphisms of $\mathbb{Q}(\sqrt{2 \varepsilon}, \sqrt{D})$. Moreover,

$$
G_{p^{n}} / G_{p^{n-1}}=\Phi_{2 p^{n}}\left(\eta, \eta^{-1}\right)=\Phi_{2 p}\left(\eta^{p^{n-1}}, \eta^{-p^{n-1}}\right)=\Phi_{p}\left(-\eta^{p^{n-1}}, \eta^{-p^{n-1}}\right) .
$$

By Lemma 1 for $p>3$

$$
4 \Phi_{p}\left(-\eta^{p^{n-1}}, \eta^{-p^{n-1}}\right)=A_{p}\left(-\eta^{p^{n-1}}, \eta^{-p^{n-1}}\right)^{2}+p B_{p}\left(-\eta^{p^{n-1}}, \eta^{-p^{n-1}}\right)^{2} .
$$

Since $A_{p}(x, y)$ is anti-symmetric, $A_{p}(-x, y)$ is symmetric and by Lemma 2, divisible by $x+y$. The quotient is of degree $\frac{p-1}{2}-1 \equiv 0(\bmod 2)$, hence, by Lemma 3 , is a polynomial in $(x+y)^{2}$ and $x y$. It follows that $A_{p}\left(-\eta^{p^{n-1}}, \eta^{-p^{n-1}}\right)^{2}$ $=2 \varepsilon w_{1}^{2}, w_{1} \in \mathbb{Q}$. Again, by lemmas 2 and $3, B_{p}\left(-\eta^{p^{n-1}}, \eta^{-p^{n-1}}\right)^{2}=2 \varepsilon p w_{2}^{2}$, $w_{2} \in \mathbb{Q}$ and (14) follows by (15) and (16). It remains to consider $p=3$. Let $\eta^{3^{n-1}}=\sqrt{2 \varepsilon} u+\sqrt{2 \varepsilon \cdot 3^{\alpha}} v$. Then

$$
\begin{aligned}
\Phi_{3}(- & \left.\eta^{3^{n-1}}, \eta^{-3^{n-1}}\right) \\
= & \left(\sqrt{2 \varepsilon} u+\sqrt{2 \varepsilon \cdot 3^{\alpha}} v\right)^{2}-\left(\sqrt{2 \varepsilon} u+\sqrt{2 \varepsilon \cdot 3^{\alpha}} v\right)\left(\sqrt{2 \varepsilon} u-\sqrt{2 \varepsilon \cdot 3^{\alpha}} v\right) \\
& +\left(\sqrt{2 \varepsilon} u-\sqrt{2 \varepsilon \cdot 3^{\alpha}} v\right)^{2} \\
= & 2 \varepsilon\left(u^{2}+\left(3^{\frac{\alpha+1}{2}} v\right)^{2}\right)=\varepsilon\left(u+3^{\frac{\alpha+1}{2}} v\right)^{2}+\varepsilon\left(u-3^{\frac{\alpha+1}{2}} v\right)^{2} .
\end{aligned}
$$

Lemma 7. In the notation of Lemma 6, for all positive integers $n$,

$$
H_{p^{n}} / \varepsilon p H_{p^{n-1}} \text { is a sum of two squares. }
$$


Proof. We have by (11) and (12)

$$
H_{p^{n}} / H_{p^{n-1}}=\Phi_{p^{n}}\left(\eta, \eta^{-1}\right)=\Phi_{p}\left(\eta^{p^{n-1}}, \eta^{-p^{n-1}}\right)
$$

and by Lemma 1 ,

$$
4 H_{p^{n}} / H_{p^{n-1}}=A_{p}\left(\eta^{p^{n-1}}, \eta^{-p^{n-1}}\right)^{2}+p B_{p}\left(\eta^{p^{n-1}}, \eta^{-p^{n-1}}\right)^{2} .
$$

Since $A_{p}(x, y)$ is anti-symmetric, $A_{p}(x, y) /(x-y)$ is symmetric of even degree and, by Lemma 3 ,

$$
A_{p}\left(\eta^{p^{n-1}}, \eta^{-p^{n-1}}\right)^{2}=2 \varepsilon p w_{3}^{2}, w_{3} \in \mathbb{Q} .
$$

Also $B_{p}(x, y) /(x+y)$ is symmetric of even degree, hence

$$
B_{p}\left(\eta^{p^{n-1}}, \eta^{-p^{n-1}}\right)^{2}=2 \varepsilon w_{4}^{2}, w_{4} \in \mathbb{Q}
$$

(17) follows from (18)-(21). It remains to consider $p=3$. Let $\eta^{3^{n-1}}=\sqrt{2 \varepsilon} u+$ $\sqrt{2 \varepsilon \cdot 3^{\alpha}} v$. Then

$$
\begin{aligned}
& F_{3^{n}} / 3 \varepsilon F_{3^{n-1}} \\
& =\frac{1}{3 \varepsilon}\left(\left(\sqrt{2 \varepsilon} u+\sqrt{2 \varepsilon \cdot 3^{\alpha}} v\right)^{2}+\left(\sqrt{2 \varepsilon} u+\sqrt{2 \varepsilon \cdot 3^{\alpha}} v\right)\left(\sqrt{2 \varepsilon} u-\sqrt{2 \varepsilon \cdot 3^{\alpha}} v\right)\right. \\
& \left.\quad+\left(\sqrt{2 \varepsilon} u-\sqrt{2 \varepsilon \cdot 3^{\alpha}} v\right)^{2}\right) \\
& =2\left(u^{2}+v^{2}\right)=(u+v)^{2}+(u-v)^{2} .
\end{aligned}
$$

Proof of Theorem 2-3. It follows by induction on $n$ from Lemmas 6 and 7 that for $n$ even $G_{p^{n}}$ and $H_{p^{n}}$ are sums of two squares. The formula (13) gives the theorems.

Open Access. This article is distributed under the terms of the Creative Commons Attribution License which permits any use, distribution, and reproduction in any medium, provided the original author(s) and the source are credited.

\section{Reference}

[1] H. Riesel, Prime Numbers and Computer Methods for Factorization, Birkhäuser 1985.

\section{A. SCHINZEL}

Institute of Mathematics,

Polish Academy of Sciences,

Sniadeckich 8,

P.O.Box 21, 00-956 Warszawa,

Poland

e-mail: schinzel@impan.gov.pl 


\section{SkaєbA}

Institute of Mathematics,

University of Warsaw,

Banacha 2,

02-097 Warszawa,

Poland

e-mail: skalba@mimuw.edu.pl

Received: 22 April 2013 